\title{
Tangence
}

\section{De Mourir de penser à Critique du jugement. Vivre. Créer. Publier}

\section{From Mourir de penser to Critique du jugement. Living. Creating. Publishing}

\section{Agnès Cousin de Ravel}

Numéro 115, 2017

Traversées de Pascal Quignard

URI : https://id.erudit.org/iderudit/1045149ar

DOI : https://doi.org/10.7202/1045149ar

Aller au sommaire du numéro

Éditeur(s)

Tangence

ISSN

1189-4563 (imprimé)

1710-0305 (numérique)

Découvrir la revue

Citer cet article

Cousin de Ravel, A. (2017). De Mourir de penser à Critique du jugement. Vivre. Créer. Publier. Tangence, (115), 143-155. https://doi.org/10.7202/1045149ar
Résumé de l'article

Les livres que Pascal Quignard publie depuis près d'un demi-siècle, éloignés de la pratique de l'autofiction, se nourrissent néanmoins des événements marquants de sa vie (naissance, sexualité précoce, emprise maternelle, ses rencontres), comme de ses lectures dans tous les domaines de la connaissance. Il lit, il écrit, il publie, partagé entre son besoin vital de solitude pour lire, pour penser, pour méditer, pour écrire, et la nécessité de réintégrer le groupe au moment de la publication. Ses livres, entre rêve et réalité, ne revendiquent aucune doctrine, ne prétendent à aucun enseignement, même s'ils ne cessent de se nourrir de notre histoire contemporaine comme de celle du passé le plus lointain. Ils sont une invitation à penser. Depuis quelques années, Pascal Quignard est sur scène, seul ou avec d'autres artistes, jouant du piano, lisant, interprétant ses textes et donnant ainsi chair à ses livres. 


\section{De Mourir de penser à Critique du jugement. Vivre. Créer. Publier Agnès Cousin de Ravel Université Paris-Diderot}

«... ce vivre-écrire que je suis»

Irène Fenoglio ${ }^{1}$

Où se tient la porte de l'autre monde? Où sont ceux que j'aime? [...] Où sont les livres, là où s'ouvre le temps?

Pascal Quignard ${ }^{2}$

\section{Passages. Seuils. Portes}

Les larmes, roman inactuel et novateur. Puissance de métamorphose de l'écriture. Premières lignes, première page. Un monde s'ouvre. Le temps n'est plus le temps:

Jadis les chevaux étaient libres. Ils galopaient sur la terre sans que les hommes les désirent, les encerclent, les regroupent dans les défilés, les prennent au lasso, les piègent, les attellent aux chars de guerre, les harnachent, les sellent, les ferrent, les montent, les sacrifient, les mangent. Parfois les hommes et les bêtes chantaient ensemble. Les longs gémissements des uns provoquaient les singuliers hennissements des autres ${ }^{3}$.

1. Titre d'un entretien avec Irène Fenoglio, Genesis, no 27, 2006, p. 97-106.

2. Pascal Quignard, Princesse vieille Reine, Paris, Galilée, 2015, p. 50-51.

3. Pascal Quignard, Les larmes, Paris, Grasset, 2016, p. 7. Désormais, les références à cet ouvrage seront indiquées par le sigle $L L$, suivi de la page, et placées entre parenthèses dans le corps du texte. 
Lire Pascal Quignard, aller de livre en livre (plus de quatre-vingts livres publiés à ce jour), s'abandonner à la puissance magique de leurs images, c'est, à sa suite, parcourir des espaces presque infinis. Depuis L'être du balbutiement paru en 1969, son œuvre multiple, singulière, foisonnante est toujours en chemin. Témoignant de sa diversité, de sa richesse et des voies multiples qu'il explore depuis plus de quarante-cinq ans, de mai 2014 à mai 2017 onze livres: Mourir de penser (le tome Ix de Dernier royaume, septembre 2014), Une vie de peintre. Marie Morel, avec Marie Morel (décembre 2014), Sur l'idée d'une communauté de solitaires et Critique du jugement (mars 2015), Princesse vieille Reine (août 2015), Vita e morte di Nitardo (Vie et mort de Nithard, janvier 2016), Les larmes (septembre 2016), Le chant du marais (octobre 2016), Performances de ténèbres (janvier 2017), Une journée de bonheur (mars 2017) et Dans ce jardin qu'on aimait (mai 2017); livres auxquels on peut ajouter trois de ses textes parus ${ }^{4}$ récemment: "Commentaire sur trois vers de Donne ${ }^{5}$ », «Le mot littérature "est d'origine encore inconnue" ${ }^{\text {» }}$ et une série de brefs entretiens avec Mireille Calle-Gruber dans Le dictionnaire sauvage. Pascal Quignard.

Passages. Le mot est ouverture, mouvement; il est aussi transformation, métamorphose. Mais le seuil est-il fermeture? Abandon d'un monde? Le point de non-retour? Est-il séparation ou lien entre le dehors et le dedans, entre réel et imaginaire? Entre le rêve et le fantasme? Entre le rêve et la réalité? Verneuil ou Bergheim? Le seuil est-il l'entrée dans l'espace de l'autre, des autres sans s'y perdre, en y prenant sa place? En y étant reconnu. Ou est-il ouverture vers un espace toujours plus vaste? Vers une initiation? "Hétérotopies", disait Foucault. De la vie fœtale à la vie aérienne, neuf métamorphoses selon Pascal Quignard, chez qui il est bien davantage question de la porosité des frontières, de passage entre le dedans et le dehors. De la vie sociale à la vie solitaire, de la vie au livre, du livre à la vie, sa pensée se tisse, s'étire, s'étend de façon aussi dense, aussi complexe qu'un réseau neuronal. Passage du sommeil à la veille, la

4. Plus d'une douzaine de textes parus dans des livres et en revues de 2014 à 2016.

5. Pascal Quignard, "L'énigme», suivi de «Commentaire sur trois vers de Donne», dans Mireille Calle-Gruber, Jonathan Degenève et Irène Fenoglio (dir.), Pascal Quignard. Translations et métamorphoses, Paris, Hermann, 2015.

6. Pascal Quignard, "Le mot littérature "est d'origine encore inconnue" ", dans Irène Fenoglio (dir.), Autour d'Émile Benveniste, Paris, Seuil, 2016. 
patte de la chatte qui perçoit le moindre frémissement. Pour Pascal Quignard, qui fait corps avec ses livres - eux en lui, lui en eux -, la sur-métamorphose mystérieuse de la création, la transmutation d'un vrai chronologique en faux apparent de l'écriture ou l'inverse, circonscrivant des univers mouvants: le moi et le rêve, l'histoire et la littérature, le critique et l'écrivain.

Être dans le groupe ou hors du groupe. Étrange communauté des Solitaires de Port-Royal, des lecteurs, des créateurs tels qu'ils apparaissent dans Les larmes: "Or, certains de ces hommes paraissaient plus étranges encore, s'il est possible. Car ils se dissociaient du groupe qui les avait éduqués et des mères qui leur avaient enseigné leur langue et qui avaient cherché à apprivoiser leur sauvagerie. Eux, ils persistaient dans le silence et ils restaient dans l'ombre» ( $L L$, p. 191-192).

Aimer la solitude. Ne plus avoir peur de sa peur. Ne pas céder sur son désir. Se séparer de sa détresse sur la page. Écrire, c'est tenir. Vivre, créer, se mettre en scène. Se retirer du groupe quand il y a urgence à créer, s'en protéger quand il devient menaçant. Mais aussi le rechercher, le solliciter par le livre publié, par le jeu sur scène.

\section{Naissance et sexualité}

Un jour, un jeune homme composa ce chant: «Je suis sorti d'une femme et je me suis retrouvé face à la mort. Où se perd mon âme la nuit?» ( $L L$, p. 8)

Sur un mode plus personnel que dans ses livres précédents, Pascal Quignard revient, dans Une vie de peintre. Marie Morel et dans Critique du jugement, sur les deux expériences précoces et proches de la naissance et des «initiations génitales», parce que l'origine qui se perpétue en nous est la sexualité, la puissance «ante», le sauvage au sens que lui donne Pascal Quignard: «Sauvage ne veut pas dire non domestiqué, féroce, cruel envers les autres, barbare par rapport à ce qui est civilisé, comme les lexicologues ont pris l'habitude de le définir. Le mot latin se décompose simplement: solus + vagus. À Rome est solivagus celui qui erre seul ${ }^{7} »$. Première expérience. Première empreinte de la sexualité dans la création. La vie de chacun d'entre

7. Pascal Quignard, «Sauvage», dans Mireille Calle-Gruber et Anaïs Frantz (dir.), Dictionnaire sauvage. Pascal Quignard, Paris, Hermann, 2016. 
nous s'inscrit dans notre corps entre deux inconnus, deux nuits qui nous hantent, deux images manquantes: la vie utérine avant la naissance; après la vie, la mort. La vie utérine se situe en un lieu à jamais inconnaissable, «dans un vieux sac avant tous les vieux sacs, dans un jadis qui était peut-être l'origine elle-même ${ }^{8} »$. Puis, c'est la naissance, «la "porte à jamais fermée" " " la première expérience, violente, brutale, impensable, non mémorisable mais prégnante: «Le corps quitte un lieu pour un autre, - une vie quitte un monde pour un autre. [...] Ce foetus devenu enfant suffoque, [...] fait l'expérience de la mort ${ }^{10}$ ». La naissance est "Heimatlosigkeit ${ }^{11}$ ». Elle est la perte sans retour du pays natal, la détresse originaire que connaît tout enfant abandonné par sa mère: "C'est la Hilflosigkeit elle-même ${ }^{12}$.»

La naissance est le passage sans retour du monde liquide au monde aérien par «le trou unique du monde; la porte qui est à la fois expulsion et transmission ${ }^{13}$ ", le franchissement d'un seuil énigmatique que matérialise à la surface du corps la cicatrice de la coupure, le nombril «pas plus gros qu'une pièce de dix centimes, [...] vestige d'un lien absolu [...]. Petit bourrelet de chair crevassée, [...] obsédante ruine d'une relation directe à un amont qui est devenu à jamais impossible à la pensée et qui n'a jamais été ouvert au langage $^{14}$.» D'où les inlassables pourquoi à jamais sans réponse de l'enfant s'appropriant le langage sur les lèvres de sa mère. Puis d'autres questions: "Y a-t-il un dedans, y a-t-il un dehors quand on naît? Quel est ce lieu? Quelle est cette pénombre? Quelle est cette région? Quel est ce monde? Où suis-je ici? [...] Quel est ce sol où je tombe? Il était une fois, il y avait un dedans: il est perdu ${ }^{15}$.» Dès lors, porté par un cur infantilis intarissable, tel Lucius découvrant Pamphile se

8. Pascal Quignard, Princesse, ouvr. cité, p. 46.

9. Pascal Quignard, "Une scène française», La nuit sexuelle, Paris, Flammarion, 2007, p. 188-189.

10. Pascal Quignard, «Commentaire sur trois vers de Donne», art. cité, p. 43-44.

11. Pascal Quignard, Vie secrète, Paris, Gallimard, coll. «Folio», 1999, p. 393. Heimatlosigkeit: littéralement «perte du chez soi, du pays natal».

12. Pascal Quignard, "Lettre à Dominique Rabaté», Europe, $\mathrm{n}^{\text {os }}$ 976-977, 2010, p. 12-14. Hilflosigkeit: littéralement: «sans secours, la détresse».

13. Pascal Quignard, «Commentaire sur trois vers de Donne», art. cité, p. 45-46.

14. Pascal Quignard et Marie Morel, Une vie de peintre. Marie Morel, Pont-Aven, édition Galerie B., 2014, p. 15. Désormais, les références à cet ouvrage seront indiquées par le sigle $V P$, suivi de la page, et placées entre parenthèses dans le corps du texte.

15. Pascal Quignard, L'origine de la danse, Paris, Galilée, 2013, p. 23. C’est Pascal Quignard qui souligne. 
mettant nue, l'écrivain a le désir de voir par le trou de la serrure et, par l'écriture, d'accéder à des espaces interdits.

Portes, grilles, barreaux, délimitations de lieux clos, séparations. Telles sont aussi les portes. Le 16 juillet 1942, à Paris le Vel d'Hiv ${ }^{16}$, huis-clos de violence et de mort. Début septembre 1944, en Bavière, à Dachau, la grille sinistre portant l'inscription "Arbeit macht frei» (le travail rend libre) s'est refermée sur Jean Bruneau, l'oncle de Pascal Quignard. Néant de la mort. La grille ne s'est ouverte pour lui que neuf mois plus tard.

Seconde expérience: les «initiations à la génitalité» (VP, p. 265), violentes, cruelles et traumatisantes, laissant une trace indélébile dans le corps de certains adolescents qui les vivent, sans qu'ils l'aient souhaité: "Ces découvertes [...] peuvent être si agressives qu'elles ne sont même pas toujours "mémorisables"» (VP, p. 265). Elles restent voilées et ne sont dicibles que dans l'après-coup de la création. Pascal Quignard les évoque dans Une vie de peintre: «Le corps s'est rebiffé, rétracté, réservé, muselé, densifié, je ne sais pas comment il faut exprimer cette singulière condensation à laquelle il a été soumis. J'ai connu cet écart [...]. Il reste fasciné en moi. Je ne suis pas libre» (VP, p. 266). Une telle expérience est une initiation, « une mystagogie. [...] une révélation [...] sans retour» (VP, p. 267). Celui ou celle qui, à son corps défendant l'a subie, est tel les faucons qui, «après qu'on a ôté le capuchon de peau qui voilait leur visage, à la suite de leur première mue, $[\ldots]$ ne sont désormais ni sauvages ni domestiqués: ils sont devenus hagards» (VP, p. 269), à entendre dans son sens étymologique: «Hagard vient de "hager" [...] Hager s'éprouve dans la violence de l'égarement ${ }^{17}$."

Ces deux expériences de la perte sont comme une plongée brutale, subie, dans un monde qui sidère. Le corps assure dès lors sa prééminence sur la pensée et la création est, en conséquence, chez Pascal Quignard, comme une enveloppe, une peau, une toile autour de ce qui a surgi, qui reconstitue «l'étrange sac spatial» (VP, p. 265) crevé par la naissance puis par des initiations précoces à la sexualité. Elle est, selon le mot du psychanalyste Didier Anzieu, l'enveloppe «autour de l'impensé, de l'innommable, de l'irreprésenté, de

16. Pascal Quignard évoque la rafle du Vel d'Hiv dans Les désarçonnés, Paris, Grasset, 2012, p. 29.

17. Pascal Quignard, La barque silencieuse, Paris, Seuil, 2009, p. 100. 
l'inéprouvable ${ }^{18}$ ». Plus encore, elle a accès «à l'accès perdu» (VP, p. 15), à la Perdue, à "ce "Contenant perdu" par excellence qui suscite les objets de l'art ${ }^{19} »$. Elle est ce que rend possible l'écriture, quand elle fait revenir quelque chose d'antérieur au langage, ce "Quelque chose qui peut remonter avant le sujet, avant la langue si longuement et si péniblement acquise, avec d'autres traces que celle de la langue ${ }^{20} »$. Ce dont Pascal Quignard fait l'expérience depuis qu'il écrit lui a été «théoriquement» confirmé quand il a lu lors de leur parution les Dernières leçons ${ }^{21}$ de Benveniste.

\section{De la création à la publication}

À la fin de son service militaire, en 1972, Pascal Quignard, "Collectionneur d'images qui font baisser les yeux ${ }^{22}$ », commence à collectionner des images érotiques qui au départ le heurtent et qui seront à la source de deux livres, Le sexe et l'effroi et sa suite, La nuit sexuelle. Ces images (par exemple la «Femme à la souricière» de Mellan, les dessins, les peintures de Courbet, de Masson, de Bellmer, de Rustin ou de Marie Morel) fonctionnent comme les images du rêve — elles sont le «nombril du rêve» (VP, p. 15; Pascal Quignard reprend le mot de Freud) —, conçu comme le mode de passage entre l'interne et l'externe, entre le nocturne et le diurne, entre le corps et la pensée ${ }^{23}$. Elles connectent le créateur à l'avant langage, au monde non verbal, non mémoriel, érotique, impulsif, sauvage de l'infantia. Elles sont «[1]a pensée en images (Denken in Bilden) [...] plus hallucinante que la délibération verbale. [...] à la fois plus ancienne que la pensée en mots et plus saisissante que la conscience qui dérive de cette dernière ${ }^{24}$ ». Tout à la fois semblables au "travail invisible» des femmes lors de la conception et un rempart, elles entrouvrent la porte que le langage, lors de son acquisition au temps de la pueritia, avait fermée, refoulant alors la question de l'origine. Cette pensée

18. Didier Anzieu, Créer. Détruire, Paris, Dunod, 1996, p. 29.

19. Pascal Quignard, Critique du jugement, Paris, Galilée, 2015, p. 241. Désormais, les références à cet ouvrage seront indiquées par le sigle $C J$, suivi de la page, et placées entre parenthèses dans le corps du texte.

20. Pascal Quignard, «Le mot littérature», art. cité, p. 270.

21. Émile Benveniste, Dernières leçons. Collège de France, 1968 et 1969, éd. JeanClaude Coquet et Irène Fenoglio, Paris, EHESS Éd./Gallimard/Seuil, 2012.

22. Pascal Quignard, «Collectionneur d'images qui font baisser les yeux», L'Infini, no 87, été 2004, p. 3.

23. Voir Pascal Quignard, La nuit sexuelle, ouvr. cité, p. 86 et 107.

24. Pascal Quignard, La nuit, ouvr. cité, p. 92. 
«par images, par débris de rêves, par motions, par é-motions, par départs, par fugues, par extases ${ }^{25} »$, qui est comme un voir dans la nuit, à «la frontière de la chair et de la pensée ${ }^{26}$ », irrigue le sabir de l'écrivain. Plus loin encore, en amont, plus proche encore de l'origine,

chez les hommes qui créent un étrange regard vient, au fond d'eux-mêmes, qui puise au fond de leur corps. Ce regard semble germer du fond de leur vie ancienne. Il vient de l'enfer en vérité. Il procède des morts, il descend en ligne directe du monde des fauves, il émerge du jadis.

Ce front qui se resserre, ces sourcils qui se rapprochent, ce silence qui se fait, cette main qui se suspend, tous se concentrent vers une unité mystérieuse.

Dans tous les cas envisagés, dans le plus complet mutisme, cette extase qui n'a pas encore ses mots, cette spéculation les yeux vides, cette oniromancie qui enquête et qui cherche, cette énigme sont délivrées ou engendrées ailleurs que chez les vivants. Elles sont tournées vers un autre monde que le monde ( $L L$, p. 107).

Soumis à l'élan qui monte en lui avec la puissance de la physis, comme la sève monte au printemps, l'œuvre monte en celui qui crée «comme la colère où l'âme va lâcher prise [...]. Elle monte comme le plaisir qui avale dans son immense vague [...]./ Nous ne pouvons pas refouler l'image qui hante» $(V P$, p. 372). Avec, pour conséquence, l'implication totale dans sa création du créateur, officiant d'un mystère parce que: «Créer c'est être à sa chose. C'est être un» (CJ, p. 49). C'est ce que Pascal Quignard nomme son " "vivrécrire"27", chacun de ses livres étant alors pour lui «le cœur de [son] cœur» (CJ, p. 230). Être à sa chose, être à soi comme Rousseau sur l'île Saint-Pierre ${ }^{28}$, se soumettre à l'urgence de créer, au risque, sinon, de voir disparaître ce qui monte en lui ( "Voilà la sensation "finir cela avant de mourir”29»),

25. Pascal Quignard, Sur l'idée d'une communauté de solitaires, Paris, Arlea, 2015, p. 13.

26. Pascal Quignard, Sur l'idée, ouvr. cité, p. 13.

27. Pascal Quignard, «Le mot littérature», art. cité, p. 270.

28. " [J]e ne suis à moi que quand je suis seul, hors de là je suis le jouet de tous ceux qui m'entourent» (Jean-Jacques Rousseau, Les rêveries du promeneur solitaire, dans Euvres complètes, éd. Bernard Gagnebin et Marcel Raymond, avec la collaboration de Robert Osmont, Paris, Gallimard, coll. «La Pléiade», 1996, t. I, p. 1094).

29. Pascal Quignard, Sur l'idée, ouvr. cité, p. 31. 
impose au créateur de se retirer du groupe, ne plus répondre à l'appel. Pascal Quignard, "dans l'impatience d'être seul ${ }^{30}$ », se sépare de ses amis, monte dans sa petite chambre sous les toits à Sens, au lieu de l'Absence, selon le mot de George Sand. Solitude voulue, nécessaire, forme d'ascétisme. Retrait en soi-même hors de la communauté pour «se perdre dans son nuage gris ou noir, sa brume, son souffle, son ombre, sa chose, son rêve, son invisible» (CJ, p. 117).

Quel que soit, ensuite, le jugement des professeurs, des critiques (univers auquel lui-même a participé pendant vingt-cinq ans) ou des jurys des prix littéraires, il lui faut créer même si le fruit de sa création est contraire à ses intérêts, car l'alternative est claire: créer ou mourir. Indifférence du créateur face au jugement et à la critique: "Pensez ce que vous voulez mais ce que vous en pensez", cela ne compte pas beaucoup à mes yeux. Il me faut écrire ces pages avant de mourir. C'est tout» (CJ, p. 229). Il lui faut créer, aller «du vide au plein. [...] du plein au vide ${ }^{31} »$. Renonçant temporairement au langage, pour faire monter en lui son propre langage, Pascal Quignard s'ouvre à l'imprévisible, à «la contemplation silencieuse $\mathrm{e}^{32}$ ». Comme «un grand lire immense $e^{33}$ ». La publication de chacun de ses livres est alors pour lui «la fin de l'hallucination, le surgissement imprévisible des avis, le retour de la «Beurteilung ${ }^{34}$ » (le jugement; CJ, p. 210).

Après avoir renoncé à faire corps avec le groupe, la bande, sa violence, son goût du sang versé et de la charogne, son plaisir de tuer, après avoir menacé sa cohésion en s'en étant retiré pour écrire, après l'avoir trahi, tel Spinoza ou Rousseau, il le réintègre temporairement. Il quitte «la solitude, les retraits, l'errance, la nuée de la création, la pénombre de l'origine pour une espèce de tourisme dans l'horreur cancanière et fiévreuse des congénères » (CJ, p. 227). Publier au risque de la mort apparaît alors comme une expiation. Pascal Quignard reprend le mot de Tchouang-tseu: «Publier livre, s'exposer supplice» (CJ, p. 204). Publier, c'est avouer;

30. Pascal Quignard, Mourir de penser, Paris, Grasset, 2014, p. 214.

31. Pascal Quignard, Mourir, ouvr. cité, p. 183.

32. Pascal Quignard, "Ce que vous a apporté Claude Simon» (postface), dans Mireille Calle-Gruber (dir.), Les triptyques de Claude Simon ou l'art du montage, Paris, Presses Sorbonne Nouvelle, 2009, p. 207.

33. Pascal Quignard, «Ce que vous a apporté Claude Simon», postface citée, p. 211. C'est Pascal Quignard qui souligne.

34. Le mot n'est pas en majuscules dans le texte. 
il faudrait éviter de se laisser prendre au piège de la parole. Déjà dans «Homologia »: «Parler est toujours excessif ${ }^{35}$ ». D'ailleurs de l'aveu ${ }^{36}$ à la mort, il n'y a qu'un pas. La Châtelaine de Vergy, Mademoiselle de Clermont, ou les victimes du Centre S21 au Cambodge en 1977 en ont fait la terrible épreuve. C'est, dit autrement: «Racine, Hamon livrés aux crocs des chiens dans la neige et le silence. Ces corps sortis de leur tombe comme une parution. Allélophagie» (CJ, p. 206).

La publication et le jugement qu'elle provoque sont à l'image du fonctionnement de toute société. C'est pour l'écrivain, contraint, parce qu'il serait encore pire pour lui de ne pas publier, s'exposer "à se "faire tuer gratis pro deo"» (CJ, p. 224). C'est le prix à payer pour la promotion du livre que désormais Pascal Quignard assure avec parcimonie.

\section{Entre désir et rêve}

Ce simulacre de mise à mort de l'écrivain lui impose de fuir. Fuir la curée sociale. Fuir les réseaux, l'organisation de l'État au risque d'attiser encore davantage la rancune de la société. Fuir le jugement qui blesse à mort tout en ravivant le désir d'être seul et d'être à nouveau libre, sans dette, sans autorisation à demander: «Ne pas se défendre; le nombre étoufferait. Ne pas hausser une voix inutile; la clameur l'engloutirait. Ne jamais se donner la détresse de se défendre mais, aussitôt après, aussitôt le jugement rendu, s'enfuir, s'évader, dérouter les poursuivants, [...] ne pas céder sur sa liberté, être sans feu ni lieu, être introuvable» (CJ, p. 122). Errer, sortir des chemins tracés, se dés-assujettir du lien social, tel Hartnid qui jamais ne s'arrêtait. Il «naviguait, chevauchait, assouvissait ses envies, ses peurs, ses dégoûts, ses hontes, à l'autre bout du monde, de l'autre côté du monde» ( $L L$, p. 83). Libre de toute attache: «Nul ne savait dire en quelle direction partait Hartnid. Personne ne savait de quoi il pouvait vivre. Il voyageait. Il voguait. Il chevauchait. Il ne restait pas en place» (LL, p. 140-141). Fuir, glisser dans le passé à la recherche

35. Pascal Quignard, «Homologia », dans Écrits de l'éphémère, Paris, Galilée, 2005, p. 61. C'est Pascal Quignard qui souligne.

36. «Dans l'aveu le maître est l'autre et l'aveu amoncelle la charge que celui qui veut obtenir l'aveu attend. L'indication que donnait Douch à son procès m'avait terrifié. Kaing Guek Eav, alias Douch, dirigeait le Centre S21 en 1977 au Cambodge. - C'est de la faute des prisonniers s'ils meurent. Ils ne savent pas qu'il ne faut jamais avouer sous la torture. Vrai ou faux, l'aveu enclenche la fermeture du dossier qui entraîne la destruction de l'individu» (CJ, p. 231). 
du «lieu d'origine, le lieu d'avant le monde, c'est-à-dire le lieu où le moi peut être absent, où le corps s'oublie ${ }^{37}$ » et se rapprocher d'un point ultime d'abandon vers lequel le corps se tend. S'ouvrir au nonespace du vide de la pensée.

Tous les après-midi, Pascal Quignard erre de rue en rue, de quartier en quartier, dans Paris et sa banlieue ${ }^{38}$. Ou dans Sens avec sa «vieille église viking de Saint-Maurice» (LL, p. 199). Les images se mêlent aux rêves. La vie et le rêve se confondent. Son errance est recherche de traces, détachement de toute attache, absence de contraintes, confiance, plénitude, liberté. Elle est une régénérescence, une mystagogie. Il erre comme Claire jusqu'à se fondre dans l'espace, jusqu'à ne plus être «un être humain ${ }^{39}$ », sans se désintéresser du monde mais, bien au contraire, en lui portant une attention plus vive, plus aiguë, plus acérée.

Pascal Quignard, enfant, nourrissait le rêve d'entrer le soir sur scène, rejoignant un grand piano noir. L'angoisse le terrassait. Il a appris à l'aimer, pénétrant presqu'en aveugle sur la scène noire, confronté "à l'impossible "retourner en arrière", à l'impossible "se cacher dans un trou de souris" ${ }^{40} »$. Qu'il s'agisse de lecture, d'écriture ou de la scène, il est question d'une même adhésion de tout le corps, d'un même lâcher prise, d'un même abandon des résistances: «Être là ne suffit pas, il faut s'y alourdir, s'épancher dans le là./Loin en amont des pas, en deçà des gestes, tomber comme au premier jour ${ }^{41}$.» À cette condition, le «livre, la scène, est un chemin perdu, vers un pays perdu. / C'est le lieu de l'attente ${ }^{42}$.»

Depuis fin 2010, Pascal Quignard est sur scène ${ }^{43}$ dans des performances qu'il imagine et crée seul ou avec d'autres artistes. De

37. Pascal Quignard, Princesse, ouvr. cité, p. 55.

38. «[D]ans les rues piétonnières, sur les voies des vélibes, dans les impasses, dans les zones, dans les friches industrielles, dans les arrière-cours des usines sans travail» (Pascal Quignard, Les désarçonnés, ouvr. cité, p. 41).

39. Pascal Quignard, Les solidarités mystérieuses, Paris, Gallimard, 2011, p. 217.

40. Pascal Quignard, «Une scène française», La nuit, ouvr. cité, p. 188-189.

41. Pascal Quignard, L'origine, ouvr. cité, p. 151.

42. Pascal Quignard, L'origine, ouvr. cité, p. 152.

43. 28 janvier 2016, à Vérone, Pascal Quignard crée le Ballet de l'origine de la langue et de la littérature françaises, "ballet imaginaire, sans danseurs, sans oiseaux». Il est seul en scène, lisant, jouant du piano, retraçant l'histoire du 
2010 à 2013, «Medea ${ }^{44}$ ", avec Carlotta Ikeda, danseuse de buto et le musicien Alain Mahé. D’avril 2012 à avril 2014, «Femme disant adieu», «récit-récital» avec la pianiste Lorenda Ramou. Le 7 novembre 2015, à l'abbaye royale de Saint-Riquier, Vie et mort de Nithard, création de Pascal Quignard et du chorégraphe Luc Petton. Pour cette performance, Pascal Quignard a appris à tenir au poing la buse «Phoenix». Le 28 janvier 2016 à Vérone, puis le 29 mars à Eu et les 14 et 15 octobre 2016 à Paris, Pascal Quignard est seul en scène pour le Ballet de l'origine de la langue et de la littérature françaises. Le 12 février 2016, «L'Oreille qui tombe», avec la plasticienne Frédérique Nalbandian. D’une immense oreille, sculptée en savon par Frédérique Nalbandian et suspendue au-dessus de l'espace de la scène, tombent des gouttes d'eau. Pascal Quignard joue au piano des mélodies inspirées des "Chants d'oiseaux» d'Olivier Messiaen, des «Notations of bird music» de Simon Pease Cheney, dont le bruit "de l'eau qui tombe goutte à goutte de la gouttière en zinc dans le fond du jardin de la cure», et des fragments de sonates de Franz Schubert. Il lit des textes qu'il a composés. Frédérique Nalbandian lit ou chante des mots, des syllabes, des bribes de phrases de Pascal Quignard. Elle lit un long texte de Franz Schubert qui dit l'abandon, l'amour déçu, le père qui rejette son fils. Leurs gestes s'accordent. Intensité de l'émotion partagée.

Le 15 mars, à la Maison de la poésie à Paris, il est à nouveau seul en scène pour la «Performance de ténèbres sur la mort et les morts de l'automne ${ }^{45}$ ». Puis, du 8 au 14 juillet 2016, au festival d'Avignon, «La rive dans le noir» avec Marie Vialle. Et, récemment, le $1^{\text {er }}$ octobre 2016, à Paris, «Nuit blanche». Comme une ritournelle semblable à celle des boites à musique de l'enfance, Pascal Quignard a composé la musique de la «Performance de ténèbres» «Pas de chœur», avec la danseuse Marie-Agnès Gillot et Laurent Derobert, interprétée à la pointe de l'île aux cygnes. Surgissement dans la nuit toute noire des danseurs improvisant, à tour de rôle, une chorégraphie éphémère tracée sur un cercle de sable. La musique ponctue chaque perfor-

Serment de Strasbourg, première trace de la langue française et du premier poème en langue française, La Cantilène de Sainte Eulalie.

44. 27 novembre 2010, création à Bordeaux de Medea. Ultime représentation le 30 avril 2013 au Havre lors du colloque international «Les lieux de Pascal Quignard».

45. En référence aux attentats du 13 novembre 2015 à Paris. 
mance et se répète inlassablement. Jusqu'à l'obsession ou la capture. À l'amont, Sens. À l'aval, Le Havre de l'enfance. Au loin, à l'arrière, la tour Eiffel scintillante.

Les mots, le texte s'incarnent, le livre devient corps. Il est une mystique. La voix, les gestes de Pascal Quignard sont ceux du servant d'un mystère. Delphes. Peu à peu, de performance en performance, son corps se délie, s'approprie l'espace, est davantage «là» dans l'espace de la scène. Dans «La rive dans le noir», Pascal Quignard s'approche de Marie Vialle, lui fait face et lui présente le masque en tissu d'une chouette, qu'elle applique sur son visage. Sur le mode de la confidence, ils échangent quelques paroles. Leurs gestes, leurs voix sont infiniment tendres. Marie Vialle prête son corps, sa voix, à la grand-mère oiseau racontant à son petit-fils comment son grandpère a été dévoré par les oiseaux pendant la guerre de 14-18. Pour la première fois, Pascal Quignard ne lit pas son texte, mais le joue vraiment. Il met en voix et incorpore la blessure de l'enfance que le rêve cicatrise; sa mère, qui ne l'avait jamais touché, lui revenant en rêve la nuit, prononce son prénom sans mordre et sans crier. Puis, Marie Vialle apporte sur scène un corbeau bien vivant qui suit un chemin tracé de graines. Pascal Quignard le prend, se penche; le corbeau marche sur son dos. Pascal Quignard, comme seuls les enfants savent le faire, joue avec lui. Le corbeau picore dans son étui à lunettes. Jeu étrange. Ce jeu. Ce vivre. Mais, «[t] out est tellement étrange sur le rivage de lumière (in luminis oras)» (LL, p. 195). Silence. Le temps est comme suspendu. Puis les applaudissements. Pascal Quignard salue son public, puis, très vite, disparaît. Lumières dans la salle. Personne ne se lève. On chuchote, on se parle. Enfin le vide se fait.

\section{Enchantement}

Enchantés. "Entre l'effroi et l'émotion ${ }^{46}$ », tels étaient ceux qui écoutaient autrefois «chanter la tête de mort merveilleuse» de Bernon l'enfant après que Marcellin, fou de jalousie de la voix de son concurrent à la chantrerie, l'eut tué en 1583. Tels sont les lecteurs du conte bref, intense, tragique Le chant $d u$ marais, qui s'inscrit sur les pages toutes noires, d'un noir de nuit, ce noir du Sexe et l'effroi ou de La nuit sexuelle. Noir du meurtre, de la mort que l'on sent au fond de

46. Pascal Quignard et Gabriel Schemoul, Le chant du marais, Paris, Chandeigne, 2016, n. p. 
soi, du chant qui s'élève du crâne de Bernon, l'enfant mort. Monde flottant, subaquatique que suggèrent les illustrations de Gabriel Schemoul. Ici ou là, une fleur, un poisson, des branchages, des fragments d'os brisés, une caisse en bois, un crâne tout blanc sur le fond noir. Dans le monde d'en haut, une souris furtive, un papillon, une libellule, une fleur. Tout se défait dans le monde des morts comme dans le rêve. Tout s'enfuit. Tout revient. Incertain et mouvant. On ne cesse de rêver en écrivant, en pensant. Dans les livres, ceux qu'on lit, ceux qu'on écrit, les rêves se mêlent aux désirs qui parfois font peur. Tels des fragments de chagrin. 